\title{
Visualization of Workaday Data Clarified by Means of Wine Fingerprints
}

\author{
Andreas Kerren \\ Linnaeus University \\ School of Computer Science, Physics and Mathematics (DFM) \\ Vejdes Plats 7, SE-351 95 Växjö, Sweden \\ kerren@acm.org
}

\begin{abstract}
More and more average users of personal computers, standard software, and web browsers come into contact with (information) visualization techniques. Depending on the task they have to perform, such visualizations are used for communication purposes, to provide a better overview of personal data, for instance pictures or emails, or to provide information of everyday commodities. After a brief outline about properties and characteristics of workaday data and their users, we focus in this paper on the visualization of wine attributes. The decision to buy a specific bottle of wine is a complex process that incorporates many different aspects from own experiences and current desires to various aromas and flavors that the wine promises to keep. We have developed two different visual representations for wine related data, which we call wine fingerprints. Both approaches are able to represent the most used wine attributes in literature and practice and can guide the purchase decision process of customers. Pros and cons of our wine fingerprints are discussed and compared with related approaches.
\end{abstract}

\section{Introduction}

Information visualization (InfoVis) for the masses, also called casual information visualization, has become an important new direction within the InfoVis community. In addition to the typical single-analyst, deep-dive analytical component of InfoVis, a growing focus of research is examining how to allow large numbers of people to produce, view and discuss information visualizations as well. The topic came up at the beginning of 2007 and was discussed during the first Dagstuhl Seminar on Information Visualization - Human-centered Issues in Visual Representation, Interaction, and Evaluation 1112 in June 2007. It was a topic of special sessions at IEEE InfoVis Conferences in 2007 and 2008 too. Several researchers work on InfoVis approaches for the masses, often with a focus on collaboration, and have developed several systems, such as the ManyEyes project [28] or the Snap-together Visualization [19].

Workaday Data: In this paper, we illuminate the fact that more and more InfoVis techniques and approaches are slowly dropping in areas where the average 
customer comes in contact with them. A lot of different commercial and opensource products were developed and are available to those people [24]. However, either these visualization systems or libraries are too complex and difficult to use or they need at least fundamental programming knowledge to apply them. However, a few approaches are intuitive and can be used and understood by non-experts without large training efforts. This is, perhaps, not the final ultimate breakthrough of the broad usage of InfoVis tools in the population. But, it is one important step in showing the "value" of information visualization [4].

Without doing a serious evaluation of this last statement, one receives the impression that mostly "simple" and single techniques are used, such as treemaps, elementary node-link drawings, tag clouds, or bubble charts 6]. ManyEyes [28] offers many such techniques, which can be used by novice users as well. This impression is not astonishing because of at least two reasons. The first one is obviously located in the simplicity of the visualization methods. They are easy to understand and to explain to casual users. Their interaction possibilities are mostly straightforward, at least from our own perspective as visualization experts. This assumption is not well researched and needs more investigation in the future. The second reason lies, in our opinion, in the visualization aesthetics and metaphor. Often, a visually appealing visualization is more successful (relating to casual users) than a more "efficient" but unappealing visualization that presents the same data. A closer look into such phenomena could improve our vague understanding of this intersection of information visualization and visualization arts. Thus, it could also improve the success of information visualization techniques in practice.

Here, we focus on the visualization of wine attributes, such as vintage, aroma, or producer. Many customers come in contact with such attributes when deciding for a specific bottle of wine. Two different visual representations for wine related data were developed in our research group. Both can be subsumed under the term wine fingerprints and should not provide any interactions in order to give us the possibility to attach them to wine descriptions in actual wine shops. They are able to represent the most used wine attributes in wine literature and daily practice and can guide the purchase decision process of customers. A further aim of our developments is to get an aesthetically pleasing result.

The remainder of this paper is organized as follows. Section 2 gives a general overview about our understanding of workaday data and its potential users. Then, we discuss our sample data set, i.e., wine attributes, and present some related approaches that offer visual representations for subsets of these attributes in Section 3. Our own approaches to visualize wine attributes by using InfoVis techniques are presented in Section 4 First evaluation results are briefly outlined in this section too. We conclude in Section 5 and give an outlook to future work.

\section{A Brief Look to Data and Users}

By workaday data, we mainly mean data that is of individual interest to one person or a smaller group/community. In InfoVis, data is typically classified by 
its type, such as the number of attributes (dimensions) per data item or structural properties (hierarchy, graph, etc.). In the following, we employ a more user-guided categorization of data into personal data and community data as proposed by J. Heer et al. 7]. We restrict our discussion on people with basic computer knowledge (writing a text, sending/receiving email messages, using web browsers, ...), but no programming or visualization experiences. Thus, our target group in this paper usually acts as a customer, and the considered visualizations are used as information and communication medium in most cases.

Personal Data: This kind of data covers various types of information, which is important for the individual, for example, own financial situation, personal collection of pictures, address books, genealogical data, inventory of hobby collectors, and much more. An additional prominent example is managing emails. Data that is related to receiving and sending emails as well as message bodies were in the focus of different research projects. A. Perer and M. Smith visualized hierarchical, correlation and temporal patterns present in individual email repositories [22. Users can identify interesting features in their repositories, e.g., how their own communication efforts look like. Another example in this context that focuses more on visualization metaphors is Anymails [8. This tool visualizes received emails by using a microbe metaphor. Here, each email is a microbe of a specific type depending on whether the sender belongs to a specific group.

Other important data for the individual is data about specific goods, such as cars or wines (if someone is interested in to buy such goods), or communal/environmental information like crime statistics in his/her surroundings. As mentioned in Section 11, we will exemplify the application of InfoVis techniques for the visualization of workaday data by means of wine attributes and properties, i.e., our focus is on personal data in this paper.

Community Data: "Examples of community data include the content of political speeches, the number of users online in a World of Warcraft realm, or voting results per county" 7. Thus, community data might be relevant for a group of persons who share similar interests or attitudes. Its importance is increasing due to the massive increase of social network applications, such as Facebook [3]. Our current work does not focus on community data.

\section{Wine Attributes and Existing Visualization Approaches}

If a consumer is buying a specific bottle of wine at the vineyard, at retailers in actual shops, or even web shops, then he/she may take different information sources into consideration for the final decision. Most people probably decide by watching the wine label on the bottle together with more or less detailed background knowledge of wines in general. Such average wine customers are interested in to know different properties or attributes of shortlisted wines, for instance, name of the wine, producer, complexity, producing country, region, grape type, color, taste, or price. Moreover, a more experienced wine consumer 
may also consider the storage type (e.g., steel cask or barrique), a rating by wine experts or other customers respectively, or the vintage of the wine. Among these properties, name, vintage, country, producer, etc. are textual attributes. The grape type, also called grape variety, is classified in two basic categories: red grapes and white grapes. Normally, red grapes are used for red wine and white grapes are used for white wine (however, a white wine can also be made from red grapes by only taking the flesh and not using their skin). The final color of a wine can be categorized into three types: red, white, and blush (rosé). In wine literature, one can find a richer color scale of about 12 different colors. All of these attributes are clear facts that can be collected from various data sources. One exception are the two most important attributes: taste and aroma. We will discuss this kind of data in Section [3.1. Note that pre-processing steps are usually needed to extract useful information from text files in idiosyncratic format. This process can be manually performed by human experts or automatically by natural language parsing techniques.

In contrast to the average wine consumer, more experienced people read wine notes (so-called tasting notes) written by wine experts, which are published in books or in the Internet. One popular source is Robert Parker's Wine Advocate 21] where tasting notes like the following one can be studied:

"The medium ruby-colored 1997 Abadia Retuerta (a blend of 65\% Tempranillo, 30\% Cabernet Sauvignon, and 5\% Merlot) exhibits an attractive spicy, cedary, tobacco, and berry fruit-scented nose. Herbaceousness makes an appearance in the mouth, but the wine is round, soft, and moderately concentrated, with fine cleanliness and accessibility. It should drink well for 5-6 years." Taken from [20].

Such tasting notes follow strict rules in their structure and diction. Even ifaccording to G. Morrot et al. 15]-humans have not developed an olfactory terminology to describe odors, there is a need to talk about different aspects of wines like smell or mouthfeel [13. How can visualization techniques help to make such textual descriptions more clear or, perhaps, to replace them? To find a general answer is not straightforward as presented in the next subsection.

\subsection{Related Works}

We present three approaches that visually represent wine attributes from various perspectives. Each related work is accompanied by a screenshot to show its aesthetics and to explain the underlying idea of aroma visualization more efficiently.

Wine Aroma Wheels. In 1984, a new standard for a common wine aroma terminology was proposed in order to facilitate the verbal communication about wine aromas [18. To support this terminology with a graphic representation, the socalled Wine Aroma Wheel was introduced by A. C. Noble et al. 17]. Meanwhile, 


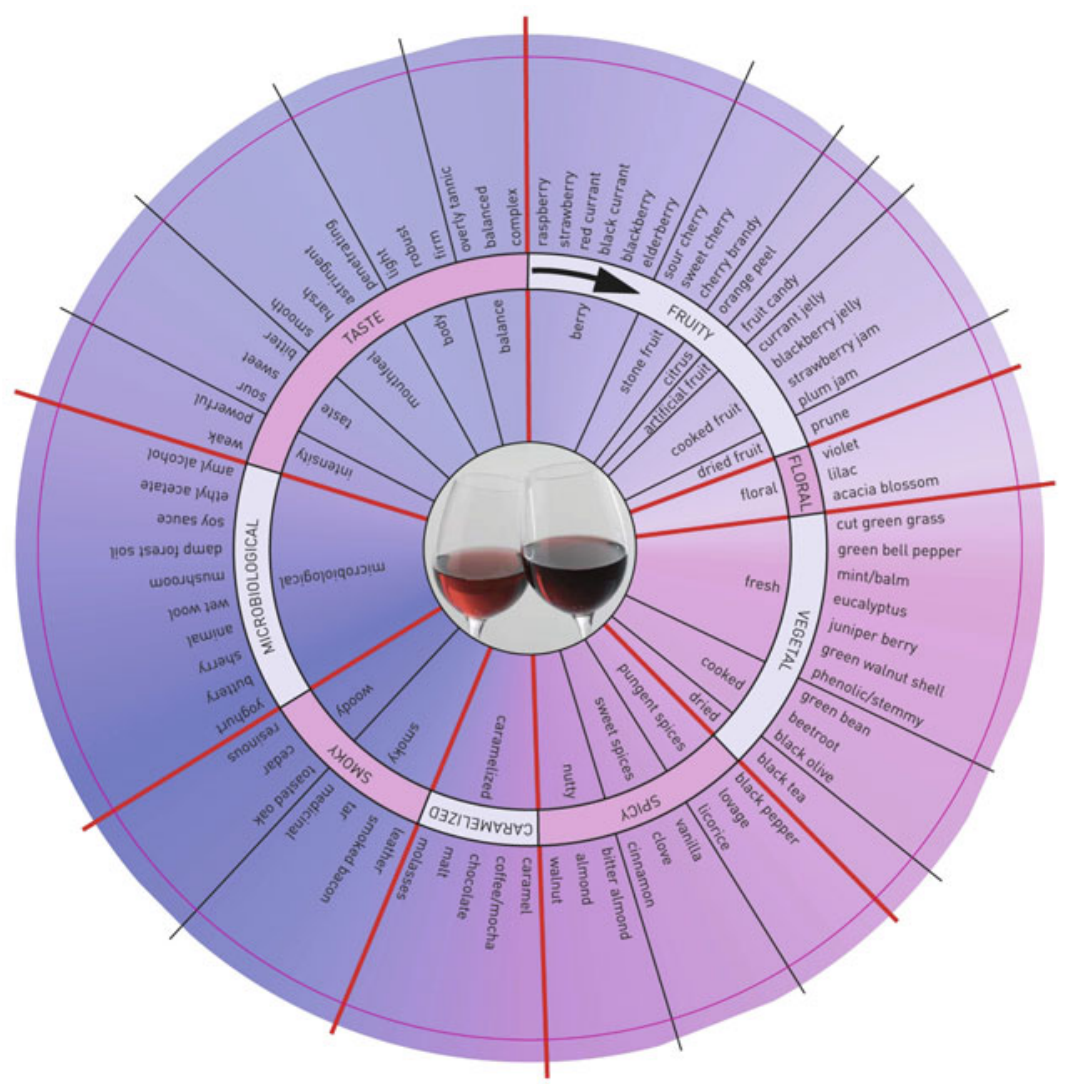

Fig. 1. Wine aroma wheel for German red wines [1. Image courtesy of Deutsches Weininstitut GmbH.

different variants of this aroma wheel came up. Fig. 11 shows a modified version for German red wines. Its hierarchical structure for different aromas is clearly to identify. For example, the more general aroma "vegetal" at the first hierarchy level can be subdivided by "fresh", "cocked" and "dried" at the next level. Then, "cooked" can be further sub-classified into "green bean", "beetroot" and "black olive" at the third level. For the wheel's practical use, the German Wine Institute writes: "The aroma wheels for German white and red wines are subdivided into colored segments that describe seven characteristic aromas and one category for taste impressions. You work your way from a broad classification in the middle to the more detailed descriptions in the outermost circle. Let's begin at "fruity". Within the broad segment "fruity", for example, your more specific impression might be that of "berries". Now, through more intense sniffing, you can determine whether your wine smells more like currants or elderberries, or in some instances, perhaps both. From the individual aromas of a wine you can draw important conclusions about its grape variety and its region of origin. [... ] Especially nice is the fact that this method of describing flavors and aromas 
enables everyone to follow experts' comments, and sooner or later, everyone can fully enjoy the pleasure of wine." [1].

For sure, such static graphics are used as a vehicle for a better communication and originally not for visualization purposes in terms of data analysis. But, the graphical representation is conceptually similar (with minor flaws due to the spatially mixed hierarchy levels) to some standard InfoVis representations for hierarchical data, such as SunBurst 25. In this way, wine aroma wheels could serve as inspiring fundamentals for further investigations in wine visualization.

Aromicon. The German company Aromicon [2] uses Flash animations [5] to visualize different attributes of specific wines as shown in Fig. 2] Each animation consists of three areas. On the top, textural information is displayed, e.g., name of the wine, vintage and origin (area). On the right hand side, some additional attributes (sweetness and tannins) are represented by icons; for example, more sugar bits are shown if the tasted sweetness is high. The rest of the image is the main part for the taste representation and the wine color. The latter one is simply represented by the background color of the image. The taste is more complex, and the different flavors and aromas are shown by using animated "real-life" icons within a wine glass moving up from the bottom of the glass. Thus, our screenshot example conveys the flavor of cherries, coffee, black pepper and cinnamon to us. Even more complicated phenomenons, such as body or sustainability of the wine, are represented by the icons' sizes and transparencies

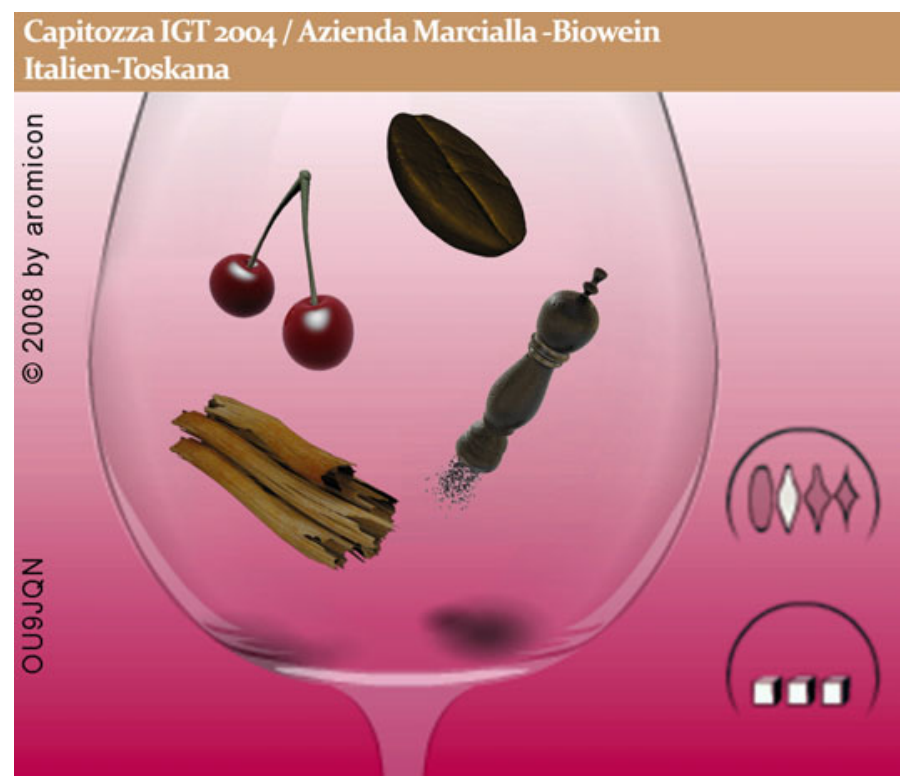

Fig. 2. Animated taste visualization of a red wine offered by the German company Aromicon [2]. Image courtesy of Aromicon GmbH. 
respectively. Aromicon applies such animations to support customers in their decision to buy wines in a web shop.

This approach is very intuitive and visually appealing. A cherry aroma is represented by a real cherry image and not by an abstract visual representation or simple text. This is obviously a strong advantage, because wine tasting is a true sensory prescription based on vision, smell, taste, and mouthfeel. Another benefit is the usage of different animation features to represent additional information. For instance, a high velocity of the animated icons is used if the wine is tangy and fresh; a low velocity stands for a more aged wine. Animation frames (or time in general) are also used to preserve the available space in small images. Conceptual drawbacks are restricted possibilities to show many different types of attributes at the same time for comparisons as well as to support more advanced customers with the aroma hierarchy discussed above.

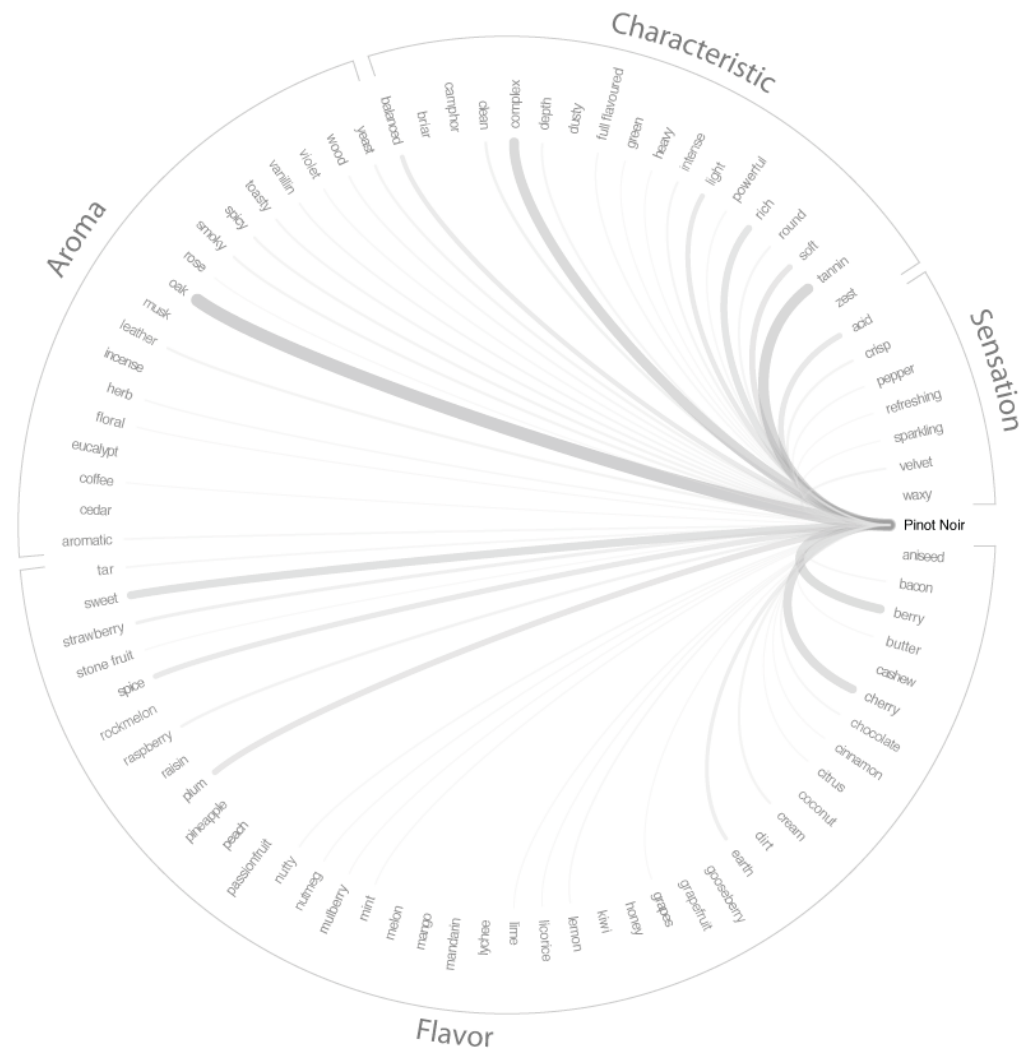

Fig. 3. Radial wine flavor visualization with regard to four different dimensions. Pinot Noir was chosen previously. Taken from the web page [26]. 
Wine Flavor Visualization. Fig. 3] shows a screenshot of a course work developed by C. Tashian at Tisch School of the Arts, New York [26]. This visualization focuses on the relationships between specific wine varieties and flavor components by using a radial hierarchical layout and arcs. The arc width represents the strength of these relationships between a wine variety itself and different attributes classified by four dimensions: sensation, characteristic, aroma, and flavor. Users can interactively choose between 11 different graphics for a small subset of varieties, i.e., no advanced interaction is provided. The underlying data set was gathered from about 5,000 wine tasting notes published over five years by a leading Australian wine magazine. In this figure, Pinot Noir was chosen as a sample wine variety. All arcs have their origin in the node on the right hand side representing this kind of wine. Thicker arcs give a hint of the most relevant properties of it. Thus, the line thickness represents the aggregated occurrences of a specific property over all tasting notes. In our case, the visualization shows that Pinot Noir has, in general, an aroma of oak with a sweet flavor of berry and cherry, a more acid sensation, etc.

This approach uses some well-known InfoVis techniques, especially a hierarchical, radial graph layout together with arcs. It is similar to M. Wattenberg's Arc Diagrams [27. Advantages are its intuitive layout and its aesthetics. It could also be used for individual wines; not only for aggregated data. However, no nominal data (e.g., producer) or more general aroma levels can be smoothly included.

\section{Visualization of Wine Attributes by Using InfoVis Techniques}

Our idea is to use space-filling and aesthetically appealing InfoVis techniques to represent a variety of attributes that are related to a specific wine 14. This data forms a multivariate data set as described in Section [3: a part of it can be hierarchically structured, such as the aroma hierarchy. Other attributes have a nominal, ordinal, or quantitative data type. For test reasons, we built up a MySQL database [16] to store a small test suite of wines together with their attributes. Currently, a wine can be attached with 14 different attributes (see Table 1). Four attributes stand out and need further explanations.

Wine Color: We distinguish between 16 different colors from Greenish Yellow to Garnet Red in ascending order. These colors are well-defined in the wine literature, such as in the Wine Advocate [21]. To prevent mistakes, we attached a unique integer ID to each color.

Rating: In practice, each professional tasting note is enhanced and complemented by a numerical rating. There are different rating systems depending on the individual wine expert or wine magazine. Parker's Wine Advocate [21] uses a 50-100 point quality scale, for example. In order to abstract from such individual systems, we restricted ourselves on an interval of integer values between 0 and 10 . This could be easily changed in the future if needed. 
Table 1. 14 wine attributes in our database together with their data type, range and visual mapping for both fingerprints (FPs). The fingerprints and their visual structures are explained in Section 4.1 and 4.2

\begin{tabular}{l||llll}
\hline Attribute & Data Type & Range & Circular FP & Rectangular FP \\
\hline \hline Name & nominal & string & circle+label & rect.+label \\
Producer & nominal & string & circle+label & rect.+label \\
Country & nominal & 13 IDs & circle+label & dot+label in a map \\
Region & nominal & any string & not used & not used \\
G. Variety & nominal & ID+string & circle+label & rect.+label \\
Complexity & nominal & 3 values & circle+color & rect.+color \\
Barrel & nominal & 2 values & circle+color & rect.+color \\
Category & nominal & 3 values & together with Color & together with Color \\
Wine Color & ordinal & 16 IDs+string circle+color+label & rect.+color+label \\
Vintage & ordinal & $1900-2008$ & circle+number & rect.+number \\
Viscosity & ordinal & any string & not used & not used \\
Rating & ordinal & $0-10$ & circle+color+size & rect.+color+size+label \\
Price & quantitative $0-1000$ SEK & circle+color+size & rect.+color+size+label \\
Aroma & hierarchical three-digit & color+balloon tree & color+treemap \\
\hline
\end{tabular}

Grape Variety: This attribute differentiates between hundreds of numbered and textually labeled grape types, whereas a negative value of a grape type number means a white grape, a positive value means a red grape. Note that up to three different grape varieties can be combined in our data set, for example, if the wine is a blend between 170 Shiraz and 35 Cabernet Sauvignon.

Aroma: According to the aroma hierarchy, each aroma attribute value consists of a list of three-digit numbers as its related tree has three levels (the root node is at Level 0), cf. Fig. 4. The 100-digit is mapped to the first level of the aroma tree, the 10-digit is mapped to the second level, and so on. For example, looking at number 521, 5 means the fifth node of the first level that is "smoky" in our data model, 2 means the second node of the second level that is "woody", 1 means the first node of the third level that is "toastedOak". It is also possible to show the first and/or the second level only by resetting the appropriate digit to 0 . Then, 500 means "smoky", for example. In this way, we can express more general and unspecified aromas, but we are theoretically restricted to nine different aroma values per level. Note that our aroma hierarchy is slightly different from the aroma wheel for German red wines in Fig. 11. The reason for this decision is that we would like to represent red and white wines, and thus, our aroma hierarchy is more generic compared to the presented one. The aforementioned restriction to nine different values is no issue in our current hierarchy.

To map this kind of data onto a visual representation, we developed two different visualizations, called wine fingerprints. They are described in the following subsections. 


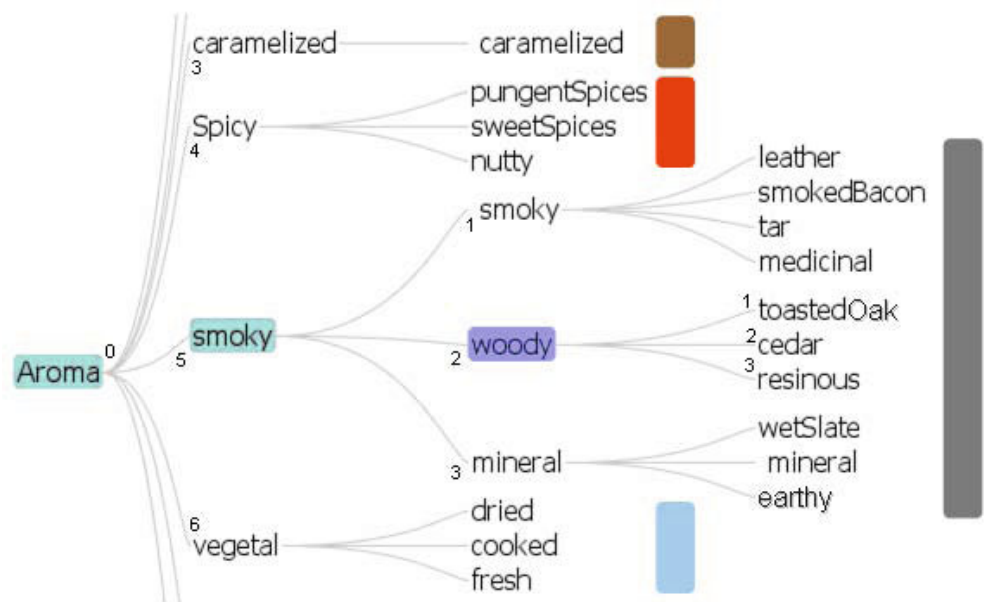

Fig. 4. A cutout of our own aroma tree structure realized in our database 14. The complete subtree of the aroma "smoky" is unfolded. The colored bars at the right hand side of the subtrees stand for the used color coding in our wine fingerprints.

\subsection{Rectangular Wine Fingerprints}

Our first approach is based on a treemap layout for representing the aroma hierarchy as shown in Fig. The most obvious difference to the second approach (see Section 4.21) is the use of a world map taken from ManyEyes 28] to represent the country attribute. It fits very well to the treemap, because they have both a rectangular shape. For space saving reasons, we decided to use only the upper and lower part of the world map according to the fact that wine grapes mostly grow between the 30th and the 50th degree of latitude in both the northern and southern hemisphere [29]. The space in between is then used for the treemap and other attribute representations.

We defined different saturated border colors and border thicknesses for the three levels of the aroma hierarchy to improve the perception of the tree structure. In the shown rectangular wine fingerprint, three different aromas and one specific taste for that specific wine are marked. The taste is colored in dark blue and corresponds to "sweet" at Level 3 of the hierarchy. The gray blue field represents "fresh" at the second level of the "vegetal" subtree etc. Remember, the deeper items are marked in the aroma hierarchy the more saturated the used colors, see the treemap area labeled with "fruity", for example.

The two map parts on the top and bottom symbolize wine-producing countries geographically. A red dot together with the country code locate the wine origin. On the right hand side besides the treemap, nine colored rectangles are used to visualize the remainder of the attributes. The vertical blue stripe displays the vintage, which is 2004 in this example. We used a slightly different representation for the vintage, because wine consumers typically have some knowledge about good and bad vintages. Thus, the pure date contains background information, 


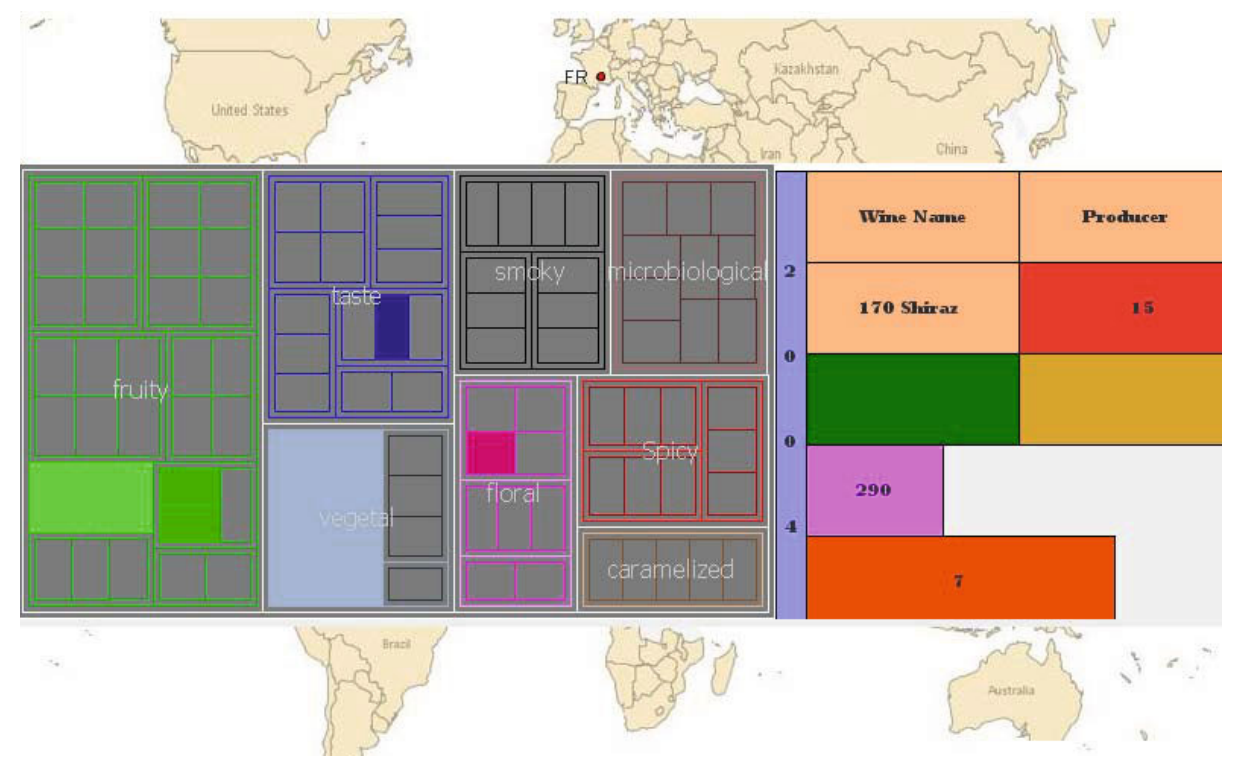

Fig. 5. A wine fingerprint using a rectangular drawing approach and a treemap layout [23] for the aroma hierarchy. The center area is divided into three rectangular areas with the treemap on the left hand side showing the complete aroma hierarchy with highlighted, individual flavors. Then, a small vertical stripe shows the vintage of the wine. On the right hand side, different areas represent nominal attributes (wine name, producer, and grape variety) by using text labels as well as ordinal/quantitative attributes (wine color, price, ....) by varying the color scale and/or widths like the two horizontal bar charts in the lower right corner. The background area indicates the origin (country) of the wine by a red dot on a world map.

which could be represented in the future too. The rectangles on the top represent from top to down and left to right the wine name, producer, grape variety (here 170 Shiraz), color \& category (white, rose and red together with the color ID; in this example, the color of the wine is 15 Brick Red), followed by complexity (dark green represents a strong complexity) and barrel type (ocher represents a wood barrel). So far, this part of the fingerprint only shows nominal and ordinal attributes. The next two rectangles are horizontal bar charts to code ordinal and quantitative data of particular importance for the customer, such as price and rating. Consequently, they are double-coded by color and size: the higher the price the longer the bar and the color is more saturated.

\subsection{Circular Wine Fingerprints}

Fig. 6] shows a screenshot example of our second approach. It mainly consists of three concentric circles plus a center point, which represents the vintage by using a text label for the year. Then, the innermost circle shows five ordinal and quantitative data items: rating results, price, wine color, complexity, and barrel 


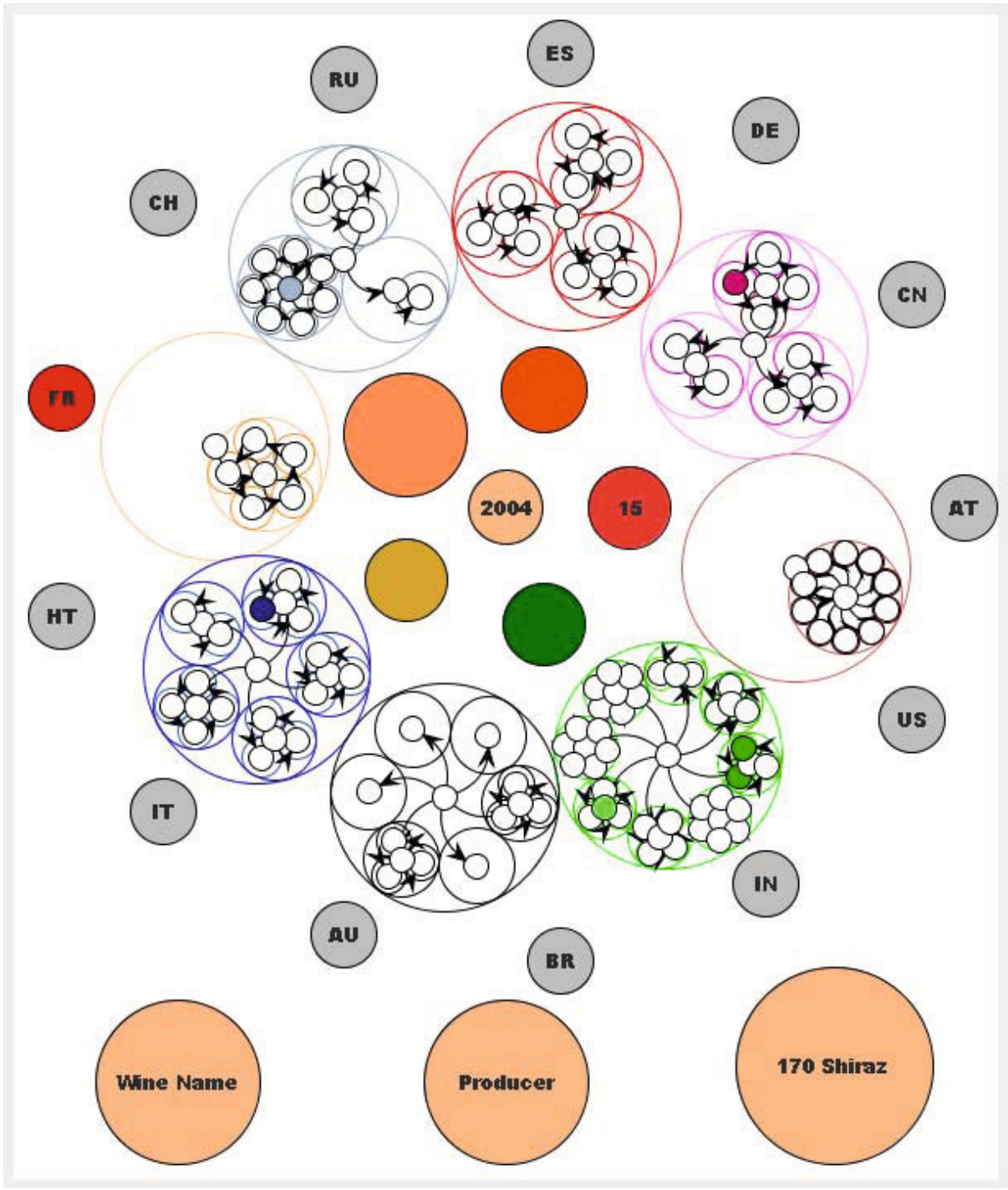

Fig. 6. Another wine fingerprint using a radial drawing approach (three layers) and a balloon tree layout for the aroma hierarchy. The midpoint of the fingerprint provides the vintage of the wine, the next circular layer represents ordinal/quantitative attributes, then the balloon tree shows the complete aroma hierarchy with marked, individual flavors. The outermost circular layer indicates the origin (country) of the wine in arbitrary order. At the bottom, nominal attributes are displayed, such as wine name, producer, and grape variety.

type. All of these attributes are double-coded in color and size or textual value respectively. The middle circle consists of a balloon tree layout (using the JUNG 
library 9] ) for displaying the complete aroma hierarchy: each subtree represents a specific, color-coded aroma/flavor class as outlined in Fig. 4. Thus, the balloon tree visualizes our aroma hierarchy in a similar way as the treemap in the previous case. Looking at the concrete fingerprint examples, the color-coding is identical for of both the rectangular and the circular approach. Those tree nodes that fit to the current wine are filled with the corresponding color. All other nodes remain unfilled. The saturation of the node coloring is increasing the deeper we are in the aroma hierarchy. Note that the current aroma selection in Fig. 6] is identical to the current aromas in Fig [5. So, the reader can easily compare both wine fingerprints. The outer ring represents a (probably incomplete) set of countries that grow wine. Each country is represented by a circle and can be identified by its international country code. Similar as before, the current country where the wine was produced is marked in red color. The three circles at the lower part of this wine fingerprint represent nominal data: name of the wine, producer, and grape type. The circle for the grape variety is larger than the ones for name and producer in order to provide more space for up to three different varieties.

\subsection{User Study and Discussion}

We decided to develop both approaches to get a better feeling of which approach is better suited for the underlying wine data set. A first small user study with ten test persons was performed and yielded valuable hints for further improvements. The test design was straightforward. At first, the test persons should learn the different graphical components of the wine fingerprints with the help of an interactive user interface to become familiar with our ideas. For instance, they could select some flavors/aromas in the GUI and verify the resulting fingerprints immediately. After this learning phase, they had to compare various predefined wine fingerprints with a fingerprint based on a specific wine selection in order to identify the best match. Finally, they got a short questionnaire with rating and open questions.

Some results were surprising, for instance that our test persons did not understand the rectangular wine fingerprints using the treemap approach very well. Here, they had problems to identify the most appropriate fingerprint in the comparison task $(62 \%$ correct answers). The results were better for the corresponding task with the circular approach (75\% correct answers). Furthermore, seven of ten subjects would prefer our circular approach. Moreover, some test persons had the impression that the various circles within the circular fingerprints are a metaphor for wine grapes, which was originally not intended by us. Of course, the results of this user study are not significant and a more detailed study must be prepared.

If we compare our approaches to those discussed in Section [3.1] then we can identify pros and cons for them. Table 2 gives an overview of differences according to a set of features. As the Aroma Wheel is by definition restricted to represent aromas, tastes and flavors, it is only limited comparable to the other approaches, which can show more information about a wine. It is obvious that Aromicon and the light-weight Wine Flavor Visualization are strong with 
Table 2. Comparison of related together with our own approaches

\begin{tabular}{l||lllll}
\hline Feature & Aroma & Wheel & Aromicon & Flavor Vis. Circular FP Rectangular FP \\
\hline \hline Accuracy & - & $\circ$ & - & ++ & ++ \\
Extensibility & -- & $\circ$ & + & ++ & ++ \\
Simplicity & + & ++ & ++ & + & $\circ$ \\
Metaphor & $\circ$ & ++ & $\circ$ & + & $\circ$ \\
Aesthetics & + & + & ++ & + & $\circ$ \\
Perception & $\circ$ & + & ++ & $\circ$ & $\circ$ \\
Intuitiveness & - & ++ & + & + & + \\
Training & - & + & + & - & - \\
Display & ++ & ++ & + & + & + \\
\hline
\end{tabular}

regard to intuitiveness, natural metaphor and aesthetics. Both cannot exactly show quantitative data and are hard to extend without destroying the overall layout. Our own approaches have their main benefit there: they are able to carry much more different types of data, and at least the Circular Fingerprint is aesthetically appealing and understandable. However, both are not easy to learn and are more difficult to perceive. One reason for this are the missing textual labels within the fingerprints to identify the graphical elements correctly. This was a conscious decision to reduce their visual complexity.

\section{Conclusions}

The aforementioned visualizations are only two examples for many applications of InfoVis techniques for workaday data. Other examples can be found in the visualization of community data [7, such as in Facebook [3] or similar services. To be successful, these visualizations must be easily perceivable, intuitive and understandable for the casual user, easy to produce in case a user plans to build an own visualization as well as follow meaningful metaphors. Our intention for this paper was not to provide a comprehensive survey on solutions for workaday data visualizations. Instead, we focused on one special application domain. We have developed two versions of wine fingerprints for specific wines and their attributes. Outgoing from a first evaluation, the circular wine fingerprint outperformed the rectangular wine fingerprint. Both approaches require some learning efforts of casual users, which is a clear drawback. Advantages are their accuracy to represent the underlying data and extensibility. We plan to perform a more detailed evaluation in the hope that the results can be used to improve the current realization of our ideas.

Describing sensory perceptions, such as vision, smell, taste, and mouthfeel, is a challenging task. Language researchers are interested in how humans talk about such sensory perceptions and what types of metaphorizations or lexical resources exist. Here, we are currently working together with Carita Paradis from the Center for Languages and Literature at Lund University. We plan to develop a visualization tool that can help to discover possible correlations or patterns 
in wine tasting databases in order to give linguists a better understanding of those (textual) descriptions. We are convinced that this research will also have a positive influence on the further development of our fingerprints.

Acknowledgments. I would like to thank Yuanxun Mei for implementing the wine fingerprint visualization as part of his Master's Thesis. Furthermore, I am thankful to Ilir Jusufi for his constructive comments that helped to develop and to improve our approach. Finally, I wish to thank Carita Paradis from the Center for Languages and Literature at Lund University, Sweden, to show us the need for visualizations of wine related data a well as to provide us with background information about this interesting field.

\section{References}

1. AromaWheel: Deutsches Weininstitut (2010), http://www.deutscheweine.de

2. Aromicon: Aromicon GmbH (2010), http://www.aromicon.com

3. Facebook: Facebook Webpage (2010), http://www.facebook.com/

4. Fekete, J.D., Wijk, J.J., Stasko, J.T., North, C.: The Value of Information Visualization. In: Kerren, et al. (eds.) [12], pp. 1-18

5. Flash: Adobe Flash (2010), http://www.adobe.com/de/products/flash/

6. Görg, C., Pohl, M., Qeli, E., Xu, K.: Visual Representations. In: Kerren, et al. (eds.) [10], pp. 163-230

7. Heer, J., van Ham, F., Carpendale, S., Weaver, C., Isenberg, P.: Creation and Collaboration: Engaging New Audiences for Information Visualization. In: Kerren, et al. (eds.) [12], pp. 92-133

8. Horn, C., Jenett, F.: Anymails Project Webpage (2007), http://www . carohorn.de/anymails/

9. JUNG: Java Universal Network/Graph Framework (2009), http://jung. sourceforge.net/

10. Kerren, A., Ebert, A., Meyer, J. (eds.): GI-Dagstuhl Research Seminar 2007. LNCS, vol. 4417. Springer, Heidelberg (2007)

11. Kerren, A., Stasko, J.T., Fekete, J.D., North, C.: Workshop Report: Information Visualization - Human-centered Issues in Visual Representation, Interaction, and Evaluation. Information Visualization 6(3), 189-196 (2007)

12. Kerren, A., Stasko, J.T., Fekete, J.D., North, C. (eds.): Information Visualization. LNCS, vol. 4950. Springer, Heidelberg (2008)

13. Lehrer, A.: Talking about Wine. Language 51(4), 901-923 (1975)

14. Mei, Y.: Visualization of Wine Attributes. Master's thesis, School of Mathematics and Systems Engineering, Växjö University, Sweden (October 2009)

15. Morrot, G., Brochet, F., Dubourdieu, D.: The Color of Odors. Brain and Languages 79(2), 309-320 (2001)

16. MySQL: An Open Source Relational Database Management System (2010), http://www.mysql.com/

17. Noble, A.C., Arnold, R.A., Buechsenstein, J., Leach, E.J., Schmidt, J.O., Stern, P.M.: Modification of a Standardized System of Wine Aroma Terminology. Am. J. Enol. Vitic. 38(2), 143-146 (1987)

18. Noble, A.C., Arnold, R.A., Masuda, B.M., Pecore, S.D., Schmidt, J.O., Stern, P.M.: Progress Towards a Standardized System of Wine Aroma Terminology. Am. J. Enol. Vitic. 35(2), 107-109 (1984) 
19. North, C., Shneiderman, B.: Snap-together Visualization: A User Interface for Coordinating Visualizations via Relational Schemata. In: AVI 2000: Proceedings of the Working Conference on Advanced Visual Interfaces, pp. 128-135. ACM, New York (2000)

20. Paradis, C.: This Beauty Should Drink Well for 10-12 Years: A Note on Recommendations as Semantic Middles. Text \& Talk 29(1), 53-73 (2009)

21. Parker, R.: Wine Advocate (2010), http://www.erobertparker.com

22. Perer, A., Smith, M.A.: Contrasting Portraits of Email Practices: Visual Approaches to Reflection and Analysis. In: AVI 2006: Proceedings of the Working Conference on Advanced Visual Interfaces, pp. 389-395. ACM, New York (2006)

23. Shneiderman, B.: Tree Visualization with Treemaps: A 2-d Space-filling Approach. ACM Transactions on Graphics 11, 92-99 (1991)

24. Shneiderman, B., Plaisant, C.: Designing the User Interface - Strategies for Effective Human-Computer Interaction, 5th edn. Pearson, London (2009)

25. Stasko, J., Zhang, E.: Focus+Context Display and Navigation Techniques for Enhancing Radial, Space-Filling Hierarchy Visualizations. In: INFOVIS 2000: Proceedings of the IEEE Symposium on Information Vizualization 2000, p. 57. IEEE Computer Society, Washington DC (2000)

26. Tashian, C.: Wine Flavor Visualization (2010), http://tashian.com/wine-flavors/

27. Wattenberg, M.: Arc Diagrams: Visualizing Structure in Strings. In: INFOVIS 2002: Proceedings of the IEEE Symposium on Information Visualization (InfoVis 2002), p. 110. IEEE Computer Society, Washington DC (2002)

28. Wattenberg, M., Kriss, J., McKeon, M.: ManyEyes: A Site for Visualization at Internet Scale. IEEE Transactions on Visualization and Computer Graphics 13(6), 1121-1128 (2007)

29. Wikipedia: List of Wine-Producing Regions (2010), http://en.wikipedia.org/wiki/List_of_wine-producing_regions 